\title{
Baseline Insulin Resistance Is a Determinant of the Small, Dense Low-Density Lipoprotein Response to Diets Differing in Saturated Fat, Protein, and Carbohydrate Contents
}

\author{
Xiuzhi Wu ${ }^{1} \mathbb{D}$, Michael A. Roussell ${ }^{2}$, Alison M. Hill ${ }^{2,3}{ }^{\circledR}$, Penny M. Kris-Etherton ${ }^{2}$ \\ and Rosemary L. Walzem ${ }^{4,5, * \text { (D) }}$ \\ 1 Department of Nutrition and Food Science, Texas A\&M University, College Station, TX 77843, USA; \\ xiuzhiwu@tamu.edu \\ 2 Department of Nutritional Sciences, Pennsylvania State University, University Park, \\ State College, PA 16802, USA; mike@mikerousell.com (M.A.R.); alison.hill@unisa.edu.au (A.M.H.); \\ pmk3@psu.edu (P.M.K.-E.) \\ 3 UniSA Clinical \& Health Sciences, University of South Australia, Adelaide, SA 5000, Australia \\ 4 Department of Poultry Science, Texas A\&M University, College Station, TX 77843, USA \\ 5 Faculty of Nutrition, Texas A\&M University, College Station, TX 77843, USA \\ * Correspondence: rosemary.walzem@ag.tamu.edu; Tel.: +1-979-847-7361
}

\section{check for}

updates

Citation: Wu, X.; Roussell, M.A.; Hill, A.M.; Kris-Etherton, P.M.; Walzem, R.L. Baseline Insulin Resistance Is a Determinant of the Small, Dense Low-Density Lipoprotein Response to Diets Differing in Saturated Fat, Protein, and Carbohydrate Contents. Nutrients 2021, 13, 4328. https:// doi.org/10.3390/nu13124328

Academic Editors: Katarzyna Przybylowicz and Giovanna Muscogiuri

Received: 26 September 2021

Accepted: 26 November 2021

Published: 30 November 2021

Publisher's Note: MDPI stays neutral with regard to jurisdictional claims in published maps and institutional affiliations.

Copyright: (c) 2021 by the authors. Licensee MDPI, Basel, Switzerland. This article is an open access article distributed under the terms and conditions of the Creative Commons Attribution (CC BY) license (https:/ / creativecommons.org/licenses/by/ $4.0 /)$.

\begin{abstract}
Individual responses to diet vary but causes other than genetics are poorly understood. This study sought to determine whether baseline values of homeostasis model assessment (HOMA-IR) was related to changes in small, dense low-density lipoprotein ( $\mathrm{sdLDL}$, i.e., $\mathrm{LDL}_{4}, \mathrm{~d}=1.044-1.063 \mathrm{~g} / \mathrm{mL}$ ) amounts quantified by isopycnic density profiling, in mildly hypercholesterolemic subjects $(n=27)$ consuming one of three low saturated fatty acid (SFA) diets: Dietary Approaches to Stop Hypertension (DASH), Beef in an Optimal Lean Diet (BOLD) and BOLD plus extra protein (BOLD+) when compared to a higher-SFA healthy American diet (HAD). The diets were consumed in random order for $5 \mathrm{wk}$, with $1 \mathrm{wk}$ between diets. BOLD+ reduced fractional abundance $(\%) \mathrm{LDL}_{4}(p<0.05)$ relative to HAD, DASH and BOLD, and reductions in $\% \mathrm{LDL}_{4}$ correlated with reductions in triglycerides $(p=0.044)$, total cholesterol $(p=0.014)$, LDL cholesterol $(p=0.004)$ and apolipoprotein B $(p<0.001)$. Responses to the four diets were similar $\left(\sim 12 \%\right.$ decrease in $\left.\% \mathrm{LDL}_{4}, p=0.890\right)$ in the lower ( $<2.73$ median) HOMA-IR subgroup but differed across diet conditions in the higher HOMAIR subgroup ( $p=0.013)$, in which $\% \mathrm{LDL}_{4}$ was reduced with BOLD+ $(-11 \%)$, was unchanged in BOLD and increased with the HAD $(8 \%)$ and DASH $(6 \%)$ diets $(p<0.05$ for BOLD+ vs. HAD). Individual responses to diet interventions are influenced by presence and degree of insulin resistance as measured by HOMA-IR.
\end{abstract}

Keywords: insulin resistance; diet response; beef consumption; saturated fat

\section{Introduction}

Low-density lipoprotein (LDL) particles are highly heterogeneous, and likely vary in atherogenicity due to differences in size, composition, and physiochemical properties. Compared with larger LDL particles, small dense LDL (sdLDL) particles have a higher propensity to penetrate the artery wall and bind to proteoglycans [1], increased susceptibility to oxidation [2] and reduced affinity for the LDL receptor [3]. Thus, a higher level of sdLDL particles is associated with increased risk for ischemic heart disease [4].

Lifestyle change to alter modifiable risk factors is often the first strategy to reduce ischemic heart disease, with reductions in total and LDL cholesterol (LDL-C) being key targets for reduction. Intervention studies demonstrate that LDL density distribution is modulated by dietary macronutrient composition. Compared with an isocaloric high-fat diet, low fat/high carbohydrate diets are associated with either no change or a decrease in LDL particle diameter (i.e., a shift toward sdLDL) [5-9]. The differences among studies 
were ascribed, in part, to differences in type (simple or complex) and proportion of energy consumed from carbohydrate (54-75\% of total energy). Replacement of carbohydrate by protein (from plant or animal sources) in moderate- or high-fat eucaloric diets is generally associated with an improved LDL density profile, with a shift away from sdLDL toward larger, more buoyant LDL subfractions $[8,10,11]$.

LDL particle diameter is related to LDL density [10]. Considerable within-study variation in individual LDL particle diameter responsiveness to diet has been consistently observed, even under controlled feeding conditions, suggesting an influence of genetic and other environmental factors. For example, apolipoprotein E phenotype [5] and sdLDL subclass pattern B [9] both have been identified as determinants of LDL responses to lower fat diets. Others have proposed that a tipping point exists where insulin resistance becomes inflammatory insulin resistance [11], and Gower et al. [12] reported that improved insulin sensitivity in response to $30 \mathrm{~g}$ of resistant starch differed depending on the subject's baseline insulin sensitivity. Further, the presence of sdLDL, or indirect indices of sdLDL, were associated with insulin resistance and diabetes in a large cross-sectional study in China [13], and with pregnancy complications in nonobese women with polycystic ovary syndrome [14].

We could find no publications reporting the effects of baseline insulin sensitivity, as judged by homeostasis model assessment (HOMA-IR), on lipoprotein density distribution response to isocaloric diets that were lower in total and saturated fatty acids (SFA) that varied in carbohydrate or protein replacement calories in subjects whose triacylglycerol, insulin and glucose values were within normal limits $[15,16]$.

The Dietary Approaches to Stop Hypertension (DASH) dietary pattern is a recommended heart healthy diet that emphasizes fruits and vegetables and is low in SFA and cholesterol $[17,18]$. Some epidemiological studies reported adverse health associations for red meat intake, which may be attributable to its SFA content, varying levels of meat processing and additives, residual confounding, or other factors [19-21]. Studies using fresh beef in interventions did not reliably show elevations in cardiovascular risk factors $[11,22,23]$. The Beef in an Optimal Lean Diet (BOLD) study compared the effects of a DASH dietary pattern with two DASH-like dietary patterns that provided different amounts of lean beef in a mildly hypercholesterolemic study sample to assess the effects of lean beef on cardiometabolic health markers in the context of healthy diet patterns [22]. The primary findings, reported previously, were the significant (and similar) reductions in total and LDL cholesterol levels in the DASH and lean beef-containing (BOLD and BOLD+) diets, compared to the healthy American control diet (HAD) [22]. In addition, the higher protein/higher lean beef BOLD+ diet was the only dietary intervention to elicit a significant reduction in systolic blood pressure compared to the control diet [23]. Samples from the BOLD study provided the opportunity to examine the effect of replacing fat calories with protein or carbohydrate calories in a well-controlled dietary setting with mildly hypercholesterolemic, but otherwise healthy subjects [22]. While the BOLD study made chemical measurements of fasting plasma lipids, it did not assess whether cholesterol reductions were associated with reductions in sdLDL. Thus, the primary aim of this secondary analysis was to assess the effects of dietary patterns that varied in the amount of protein provided by lean beef on lipoprotein density distributions, with an emphasis on changes in the abundance of sdLDL. In addition, inflammatory status and insulin sensitivity have been associated with changes in lipoprotein particle diameter in prior studies [24]. Therefore, secondary aims were to assess whether inflammatory status and insulin sensitivity at baseline were related to lipoprotein density distribution response to the different diets.

\section{Materials and Methods}

\subsection{Participants and Design}

The present study is a secondary analysis, conducted in 2014, using all 27 of the complete serum sample sets remaining from the original BOLD study [22]. Lack of a complete sample set was the sole exclusion criteria for the secondary analysis. The experimental 
design and measurements of the original study were described in detail previously [22]. Briefly, a 4-period, randomized, cross-over, controlled-feeding design was used. Participants consumed each of four diets: HAD, DASH, BOLD and BOLD+ for 5 weeks in a random order in a controlled-feeding setting with a minimum of a 1-wk compliance break between the diets. The macronutrient and fatty acid distributions of the four test diets are shown in Table 1. At the beginning of the study and at the end of each diet period, serum samples were taken from healthy men $(n=10)$ and women $(n=17)(36-65$ years of age) with moderately high baseline LDL-C ( $>2.8 \mathrm{mmol} / \mathrm{L})$. Participants were not on any lipid-lowering medications or dietary supplements during the study. Insulin resistance was not a factor in subject selection. Serum glucose and insulin concentrations were available for 25 of the 27 subjects.

Table 1. Energy and macronutrient profiles of the BOLD study diets.

\begin{tabular}{lcccc}
\hline & HAD & DASH & BOLD & BOLD+ \\
\hline 95\% Lean beef (g/day) & 20 & 28 & 113 & 153 \\
Calories (kcal) & 2097 & 2106 & 2100 & 2104 \\
Protein (\%E) & 17 & 18 & 19 & 27 \\
Carbohydrate (\%E) & 50 & 55 & 54 & 45 \\
Fat (\%E) & 33 & 27 & 28 & 28 \\
SFA (\%E) & 12 & 6 & 6 & 6 \\
MUFA (\%E) & 11 & 9 & 11 & 12 \\
PUFA (\%E) & 7 & 8 & 7 & 7 \\
\hline
\end{tabular}

The HAD was higher in SFA and total fat than the other three test diets. It also included more refined grains, added sugars, and full-fat dairy products compared to the test diets. The DASH, BOLD and BOLD+ diets included more whole grains, vegetables, fruits, and low-fat dairy products. The DASH and BOLD diets were matched for macronutrient energy distribution, but differed in the amounts of beef included, $28 \mathrm{~g} / \mathrm{d}$ and $113 \mathrm{~g} / \mathrm{d}$ lean beef respectively. The BOLD+ diet contained similar proportions of total and saturated fat as the BOLD and DASH diets but contained less carbohydrate ( $45 \%$ vs. $54-55 \%$ energy, respectively) and greater amounts of lean beef $(153 \mathrm{~g} / \mathrm{d})$ and total protein $(27 \%$ vs. $18-19 \%$ energy, respectively). The total energy content of the test diets was individually adjusted to keep participants' weight stable during the 4 feeding periods. In the original study, participants consumed one meal per weekday in the Penn State Metabolic Diet Study Center, with the other meals being prepared and packed for off-site consumption. Diet adherence was monitored by daily and weekly compliance questionnaires.

\subsection{Laboratory Assessment}

Coded samples were received from the Penn State laboratory and analyzed for lipoprotein density distribution prior to revelation of treatment codes and statistical analysis. Analytical methods for clinical chemistry and immunoassays were published in detail previously [22]. Briefly, lipid concentrations were measured enzymatically at the MS Hershey Medical Center General Clinical Research Center (Hershey, Derry, PA, USA); this same core facility measured insulin by radioimmunoassay using ${ }^{125}$ I-labeled human insulin and human insulin antiserum [25] and glucose by an immobilized enzyme biosensor for glucose [26]. Apolipoprotein B was measured by immunoturbidimetric assay at the Oklahoma Research Institute (Oklahoma City, OK, USA) under the supervision of Petar Alaupovic. High-sensitivity CRP (hsCRP) was measured with the use of latex-enhanced immunonephelometric assay (Quest Diagnostics, NJ, USA).

Insulin resistance (inversely related to insulin sensitivity) was estimated by HOMAIR [27] calculated from previously measured serum glucose and insulin concentrations using the following equation:

$$
\text { HOMA }- \text { IR }=\frac{\text { Glucose } \times \text { Insulin }}{22.5}
$$


Glucose and insulin are expressed in $\mathrm{mmol} / \mathrm{L}$ and $\mathrm{mU} / \mathrm{L}$, respectively.

Lipoprotein density profile was determined by imaging of fluorescently stained lipoproteins following NaBiEDTA ultracentrifugation as previously described with modifications [28]. Briefly, $6 \mu \mathrm{L}$ serum was incubated with $10 \mu \mathrm{L} 1 \mathrm{~g} / \mathrm{L}$ 6-((N-(7-Nitrobenz-2Oxa-1,3-Diazol-4-yl)amino)hexanoyl) Sphingosine (i.e. NBD-C6-ceramide), and $1184 \mu \mathrm{L}$ $0.18 \mathrm{~mol} / \mathrm{L}$ NaBiEDTA density gradient solution. This solution $(1150 \mu \mathrm{L})$ was then transferred to an $11 \mathrm{~mm} \times 34 \mathrm{~mm}$ thickwall polycarbonate tube and centrifuged in a Beckman Optima MAX-XP centrifuge equipped with an MLA-130 rotor (Beckman Coulter, Inc. Brea, CA, USA) for $6 \mathrm{hr}$ at $4{ }^{\circ} \mathrm{C}$. Following gentle overlayment of hexane, the tubes were immediately imaged by a CCD camera (Quantifire XI, Optronics, Muskogee, OK, USA) with a Fiber-Lite Illuminator (MH100A, Edmund Industrial Optics, Barrington, NJ, USA) as light source. The tube holder, digital camera and illuminator were positioned orthogonally to each other on an optical bench. The respective filters (Semrock, Rochester, NY, USA) were chosen to match NBD excitation (465 nm/60 nm bandwidth, part \# FF01-460-6025 ) and emission (>500 nm/long pass, part \# BLP01-488R-25) wavelengths, respectively. Pixel values of the center of the tube were converted into fluorescent intensity using Origin 7.5 (OriginLab Ltd., Northampton, MA, USA) software and plotted as a function of the tube coordinate. A total of 10 lipoprotein subclasses were identified by their density intervals $[29,30]$, and quantified by calculation of the area under the curve (AUC), i.e., pixel value. The major lipoprotein subclasses were triacylglycerol-rich lipoproteins (TRL; $\mathrm{d}<1.019 \mathrm{~g} / \mathrm{mL}), \mathrm{LDL}_{1}(\mathrm{~d}=1.019-1.023 \mathrm{~g} / \mathrm{mL}), \mathrm{LDL}_{2}(\mathrm{~d}=1.023-1.034 \mathrm{~g} / \mathrm{mL}), \mathrm{LDL}_{3}$ $(\mathrm{d}=1.034-1.044 \mathrm{~g} / \mathrm{mL}), \mathrm{LDL}_{4}(\mathrm{~d}=1.044-1.063 \mathrm{~g} / \mathrm{mL}), \mathrm{HDL}_{2 \mathrm{~b}}(\mathrm{~d}=1.063-1.091 \mathrm{~g} / \mathrm{mL})$, $\mathrm{HDL}_{2 \mathrm{a}}(\mathrm{d}=1.091-1.110 \mathrm{~g} / \mathrm{mL}), \mathrm{HDL}_{3 \mathrm{a}}(\mathrm{d}=1.110-1.133 \mathrm{~g} / \mathrm{mL}), \mathrm{HDL}_{3 \mathrm{~b}}(\mathrm{~d}=1.133-1.156 \mathrm{~g} / \mathrm{mL})$ and $\mathrm{HDL}_{3 \mathrm{c}}(\mathrm{d}=1.156-1.179 \mathrm{~g} / \mathrm{mL})$ [31]. NBD-C6-ceramide only fluoresces in a hydrophobic environment. Thus, the fluorescent intensity of a particle depends on the quantity of the hydrophobic group/molecules in lipoprotein particles and should correlate to chemical determinations of lipids. Isopycnic density profiling used here defines sdLDL as $\mathrm{LDL}_{4}$ $(\mathrm{d}=1.044-1.063 \mathrm{~g} / \mathrm{mL})$. The density profiling methodology was validated by measuring intra- and inter- assay reproducibility of samples differing in triacylglycerol (TG) concentration. Participants were grouped into quintiles based on serum TG concentration. Three serum samples from each of the $1 \mathrm{st}, 3 \mathrm{rd}$, 5 th quintiles were randomly selected and pooled and used as low $(0.63 \mathrm{mmol} / \mathrm{L})$, medium $(1.15 \mathrm{mmol} / \mathrm{L})$ and high $(2.25 \mathrm{mmol} / \mathrm{L})$ TG concentration samples. For intra-assay variation assessment, 3 replicates for each of the low, medium, and high TG samples were analyzed in a single run. For inter-assay variation assessment, 5 replicates of the samples were run on 5 separate days. On each day, a set of low, medium, and high TG samples was analyzed.

\subsection{Statistics}

Statistical analysis was performed using JMP 10.0 (SAS Institute Inc., Cary, NC, USA). Distributions were assessed for normality using the Shapiro-Wilk test. Continuous data with a skewed distribution were log transformed before analysis. Baseline characteristics between men and women were compared using two-sample t-tests. Further measurement of lipoprotein subclass by NBD-C6 ceramide distribution was compared with conventional chemical measurements from Roussell et al. [22] (see Tables 2 and 3 of this report for those chemical values) using Pearson correlation. Forest plots were determined to visualize data prior to further statistical analysis (Appendix A Figure A2). The lipoprotein density distribution and HOMA-IR at baseline and after each of the four test diets were compared by repeated measures analysis of covariance with adjustment for age, gender, and BMI, followed by Tukey-Kramer test. The primary outcomes were the differences in absolute and fractional abundance of $\mathrm{LDL}_{4}$ after the consumption of the BOLD+ diet compared to the HAD diet. Pearson correlation coefficients were calculated for the BOLD+ diet induced change in $\mathrm{LDL}_{4}$ and changes in previously reported lipid variables and apolipoprotein concentrations. To examine the effects of baseline lipids, inflammation indicators and HOMA-IR on $\mathrm{LDL}_{4}$ response to test diets, Pearson correlation coefficients were calculated 
for baseline TG, total cholesterol (TC), LDL-C, HDL cholesterol (HDL-C), HOMA-IR, and $\mathrm{CRP}$ with percentage change of $\mathrm{LDL}_{4}$ from baseline for each test diet. When a significant correlation was observed for baseline HOMA-IR and $\mathrm{LDL}_{4}$ percentage change, a secondary analysis was performed to better characterize the effect of baseline HOMA-IR. Participants with baseline HOMA-IR below the median were classified as a lower HOMA-IR subgroup $(n=13)$ whereas participants with HOMA-IR equal or greater than the median were classified as a higher HOMA-IR subgroup $(n=12)$. To investigate the responsiveness to test diets within each baseline HOMA-IR subgroup, mixed model repeated measures analysis of covariance was performed with participants entered as a random variable and age, gender, and BMI as covariates. When a significant overall dietary effect was detected by the mixed model, the Tukey-Kramer test was used to further investigate the pairwise differences between diets. The level of significance was $p<0.05$. Values for continuous variables were expressed as mean $\pm \mathrm{SEM}$.

Table 2. Baseline characteristics of study participants.

\begin{tabular}{lccc}
\hline & Men $(\boldsymbol{n}=\mathbf{1 0})$ & Women $(\boldsymbol{n}=\mathbf{1 7})$ & Total $(\boldsymbol{n}=\mathbf{2 7})$ \\
\hline Age (years) & $50.9 \pm 2.6$ & $50.9 \pm 2.2$ & $50.9 \pm 1.7$ \\
BMI & $26.5 \pm 0.8$ & $25.1 \pm 0.9$ & $25.6 \pm 0.6$ \\
TG (mmol/L) & $1.08 \pm 0.10$ & $1.10 \pm 0.08$ & $1.09 \pm 0.06$ \\
TC $(\mathrm{mmol} / \mathrm{L})$ & $4.98 \pm 0.15$ & $5.84 \pm 0.18^{*}$ & $5.52 \pm 0.15$ \\
LDL-C (mmol/L) & $3.26 \pm 0.13$ & $3.89 \pm 0.14^{*}$ & $3.65 \pm 0.12$ \\
HDL-C (mmol/L) & $1.23 \pm 0.07$ & $1.45 \pm 0.10$ & $1.37 \pm 0.07$ \\
ApoB (mg/dL) & $89.0 \pm 2.73$ & $97.9 \pm 1.82^{*}$ & $94.5 \pm 8.46$ \\
Glucose (mmol/L) & $4.94 \pm 0.08$ & $4.50 \pm 0.07$ & $4.68 \pm 0.05$ \\
Insulin (mU/L) & $12.70 \pm 1.50$ & $13.20 \pm 0.70$ & $13.00 \pm 0.70$ \\
HOMA-IR & $2.79 \pm 0.34$ & $2.65 \pm 0.16$ & $2.71 \pm 0.17$ \\
hsCRP (mg/L) & $1.00 \pm 0.16$ & $1.09 \pm 0.22$ & $1.06 \pm 0.15$ \\
\hline
\end{tabular}

Data are expressed as mean \pm SEM. ${ }^{*} p<0.05$ vs. men. Skewed data were log transformed before statistical analysis.

Table 3. Effect of diets on clinical and biochemical parameters.

\begin{tabular}{lcccc}
\hline & HAD $(\boldsymbol{n}=\mathbf{2 7})$ & DASH $(\boldsymbol{n}=\mathbf{2 7})$ & BOLD $(\boldsymbol{n}=\mathbf{2 7})$ & BOLD+ $(\boldsymbol{n}=\mathbf{2 7})$ \\
\hline BMI & $24.5 \pm 0.70$ & $25.3 \pm 0.60$ & $25.5 \pm 0.60$ & $25.4 \pm 0.60$ \\
TG $(\mathrm{mmol} / \mathrm{L})$ & $1.02 \pm 0.07$ & $1.04 \pm 0.06$ & $1.01 \pm 0.06$ & $0.98 \pm 0.06$ \\
TC $(\mathrm{mmol} / \mathrm{L})$ & $5.26 \pm 0.16$ & $5.01 \pm 0.16^{*}$ & $5.04 \pm 0.17^{*}$ & $4.96 \pm 0.16^{*}+$ \\
LDL-C $(\mathrm{mmol} / \mathrm{L})$ & $3.51 \pm 0.12$ & $3.30 \pm 0.11^{*}$ & $3.34 \pm 0.13^{*}$ & $3.29 \pm 0.12^{*}$ \\
HDL-C $(\mathrm{mmol} / \mathrm{L})$ & $1.28 \pm 0.06$ & $1.19 \pm 0.06^{*}$, & $1.22 \pm 0.06^{*}$ & $1.20 \pm 0.06^{*}+$ \\
ApoB $(\mathrm{mg} / \mathrm{dL})$ & $95.1 \pm 2.8$ & $92.2 \pm 2.8$ & $92.0 \pm 3.5$ & $91.8 \pm 2.9$ \\
Glucose $(\mathrm{mmol} / \mathrm{L})$ & $4.83 \pm 0.08$ & $4.75 \pm 0.07$ & $4.83 \pm 0.07$ & $4.91 \pm 0.08$ \\
Insulin $(\mathrm{IU} / \mathrm{mL})$ & $12.8 \pm 0.8$ & $12.3 \pm 0.7$ & $13.6 \pm 0.8$ & $13.4 \pm 0.7$ \\
HOMA-IR & $2.76 \pm 0.20$ & $2.64 \pm 0.14$ & $2.93 \pm 0.17$ & $2.94 \pm 0.17$ \\
hsCRP $(\mathrm{mg} / \mathrm{L})$ & $1.07 \pm 0.25$ & $0.99 \pm 0.19$ & $0.92 \pm 0.13$ & $0.93 \pm 0.12$ \\
\hline
\end{tabular}

Data are expressed as mean \pm SEM. $n=25$ for HOMA-IR; $n=24$ for CRP. ${ }^{*} p<0.05$ vs. baseline; ${ }^{\dagger} p<0.05$ vs. HAD (Tukey HSD). Skewed data were log transformed before statistical analysis.

\section{Results}

\subsection{Baseline Characteristics}

Baseline characteristics of the 27 participants in the original BOLD study [22] for whom complete sample sets were available are shown in Table 2. The participants were mildly hypercholesterolemic but were within the normal range for TG $(<1.7 \mathrm{mmol} / \mathrm{L})$ and HDL-C ( $>1.04 \mathrm{mmol} / \mathrm{L}$ for men and $>1.30 \mathrm{mmol} / \mathrm{L}$ for women). Baseline serum glucose and insulin concentrations did not exceed normal limits $[15,16]$.

Females had a significantly higher average TC $(p=0.003)$ and LDL-C $(p=0.007)$ than males. No differences were observed for TG, HDL-C, CRP and HOMA-IR between men and women. Despite the difference in total LDL-C concentration between men and women, 
the difference was mainly due to the presence of more large and medium LDL, but not $\mathrm{LDL}_{4}$, in women (Appendix A Figure A1). No significant gender effect on $\mathrm{LDL}_{4}$ response to the test diets was observed. Baseline HOMA-IR values varied from 1.27 to 4.73 with a median of 2.73 .

\subsection{Post-Diet Characteristics}

Table 3 summarizes previously reported [22] clinical and biochemical parameters used in lipoprotein density distribution correlation analyses. Mean values for BMI, TG, apolipoprotein B (ApoB), HOMA-IR and CRP were similar across dietary treatments. TC was reduced compared to baseline by DASH, BOLD and BOLD+; only BOLD+ reduced TC compared to HAD. Values for LDL-C were reduced by DASH, BOLD and BOLD+ when compared to baseline; no differences were observed compared to HAD. All diets lower in total and SFA reduced HDL-C compared to baseline, and both DASH and BOLD+ reduced HDL-C compared to HAD.

\subsection{Lipoprotein Density Distribution}

The AUC of core-lipid rich TRL, LDL and $\mathrm{HDL}_{2}$ were strongly correlated with chemically determined values for serum TG, LDL-C and HDL-C respectively $(r \geq 0.66, p<0.0001$ for all, Figure $1 \mathrm{~A}-\mathrm{C})$. For cholesterol-poor $\mathrm{HDL}_{3}$, there was a significant, but weaker association with HDL-C $(r=0.27, p=0.007$, Figure 1D). The lipoprotein density distribution demonstrated that technical variations across different lipoprotein subclasses were similar for samples containing differing amounts of TG, with the highest variation occurring at the top and bottom of the regions of the tube (i.e., TRL, LDL 1 , and HDL 3 c subclasses). The increase in variability was likely due to the specific solution redistribution condition at these positions during centrifuge deceleration. For other lipoprotein subclasses, the average coefficient of variation (CV) was as low as 5\% with 3 replicates for intra-assay assessments and $4 \%$ with 5 replicates for inter-assay assessments (Appendix A Table A1). Compared with baseline, no absolute or fractional change was observed for any lipoprotein subclass after the consumption of the HAD diet (Table 4). These observations support the conclusion that the macronutrient distribution of the participants' baseline diet was similar to HAD.

While the lack of differences in lipoprotein density distributions observed during baseline and HAD periods lead to the conclusion that the macronutrient compositions of baseline and HAD were similar, statistical comparisons were made relative to outcomes from the HAD controlled feeding period. Compared with the control, HAD diet, BOLD+ significantly reduced total $\mathrm{LDL}_{4}$ AUC by $\sim 9.2 \%$ which also caused a reduction in the fractional abundance $(\%)$ of $\mathrm{LDL}_{4}(p<0.05)$, indicating a shift in LDL particle diameter distribution towards larger sizes. Further analysis demonstrated that when participants consumed the BOLD+ dietary pattern, the induced decreases in $\% \mathrm{LDL}_{4}$ paralleled the decreases in TC $(r=0.48, p=0.014)$, LDL-C $(r=0.55, p=0.004)$ and ApoB $(r=0.68, p<0.001)$, with the strongest association observed for ApoB. Despite the lack of a significant difference in mean TG after the consumption of the HAD vs. the BOLD+ diet, we observed a positive correlation between the change in TG and $\mathrm{LDL}_{4}(r=0.41, p=0.044)$. The BOLD+ diet did not result in decreases in the AUC of larger diameter $\mathrm{LDL}_{2}$ and $\mathrm{LDL}_{3}$ particles, while both DASH and BOLD decreased the AUC of these LDL subclasses by an average of $\sim 4.6 \%$ and $\sim 8.8 \%$, respectively. Total $\mathrm{HDL}_{2 \mathrm{~b}}$ AUC was significantly decreased by $\sim 5.6 \%$ and $\sim 6.8 \%$ after the consumption of the DASH and BOLD diets, respectively $(p<0.05)$, but not after consumption of the BOLD+ diet. The fractional abundance of $\mathrm{HDL}_{3 a}$ increased following consumption of all three test diets low in SFA (i.e., DASH, BOLD and BOLD+) compared to the proportion present following consumption of the $\operatorname{HAD}(p<0.05)$. 
TRL

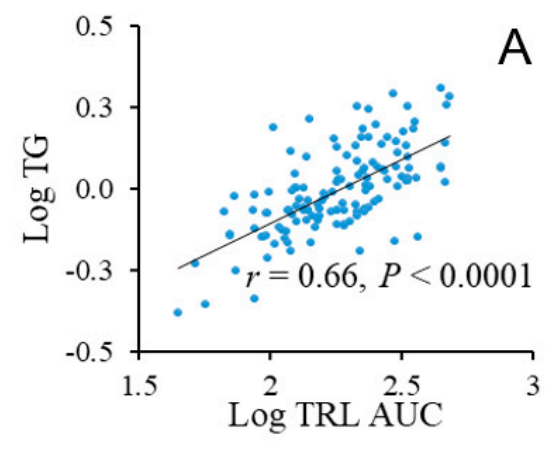

$\mathrm{HDL}_{2}$

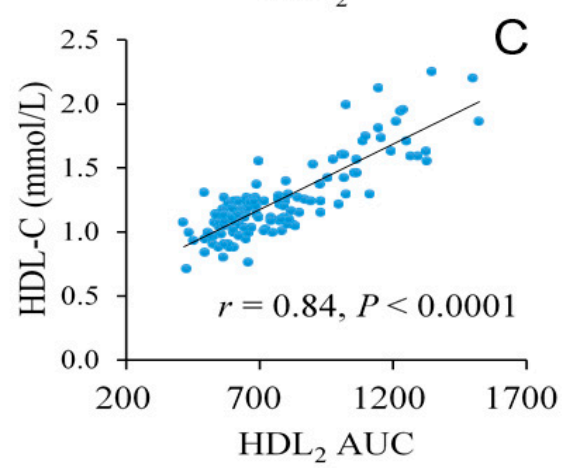

LDL

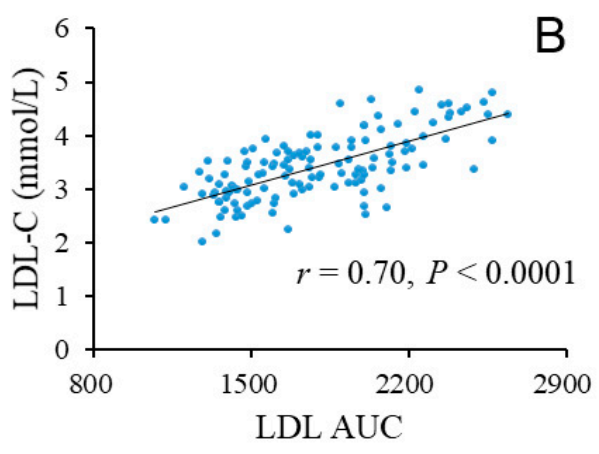

$\mathrm{HDL}_{3}$

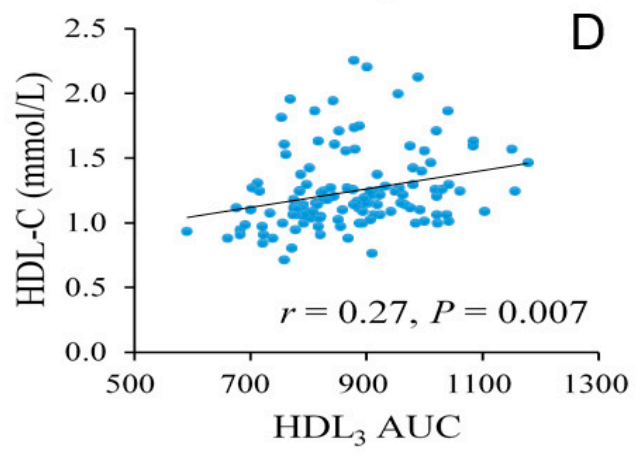

Figure 1. Correlations between lipoprotein density profile and biochemical measurements in 135 samples. Correlations are shown for TRL AUC with TG (A), LDL AUC with LDL-C (B), HDL2 AUC with HDL-C (C), $\mathrm{HDL}_{3}$ AUC with HDL-C (D). Linear regression, correlation coefficient and $p$ value are shown for each comparison. TG and TRL AUC data were log transformed to achieve normality.

Table 4. Lipoprotein density distribution at baseline and after the test diets.

\begin{tabular}{|c|c|c|c|c|c|}
\hline & $\begin{array}{c}\text { Baseline } \\
(n=27)\end{array}$ & $\begin{array}{c}\text { HAD } \\
(n=27)\end{array}$ & $\begin{array}{c}\text { DASH } \\
(n=27)\end{array}$ & $\begin{array}{c}\text { BOLD } \\
(n=27)\end{array}$ & $\begin{array}{l}\text { BOLD+ } \\
(n=27)\end{array}$ \\
\hline \multicolumn{6}{|c|}{ Lipoprotein distribution calculated as absolute AUC } \\
\hline TRL & $216 \pm 16$ & $204 \pm 22$ & $214 \pm 14$ & $181 \pm 14$ & $195 \pm 23$ \\
\hline $\mathrm{LDL}_{1}$ & $56 \pm 5$ & $44 \pm 3$ & $47 \pm 4$ & $45 \pm 4$ & $52 \pm 5$ \\
\hline $\mathrm{LDL}_{2}$ & $274 \pm 15$ & $240 \pm 15$ & $232 \pm 14$ * & $226 \pm 14 *$ & $253 \pm 21$ \\
\hline $\mathrm{LDL}_{3}$ & $780 \pm 49$ & $730 \pm 47$ & $665 \pm 48 *$ & $667 \pm 48 *$ & $687 \pm 38$ \\
\hline $\mathrm{LDL}_{4}$ & $844 \pm 39$ & $822 \pm 35$ & $797 \pm 21$ & $761 \pm 21$ & $723 \pm 33^{*,+}$ \\
\hline $\mathrm{HDL}_{2 b}$ & $369 \pm 28$ & $370 \pm 28$ & $337 \pm 22 *,+$ & $331 \pm 22 *,+$ & $345 \pm 29$ \\
\hline $\mathrm{HDL}_{2 \mathrm{a}}$ & $438 \pm 22$ & $422 \pm 24$ & $406 \pm 20$ & $408 \pm 20$ & $420 \pm 26$ \\
\hline $\mathrm{HDL}_{3 \mathrm{a}}$ & $422 \pm 12$ & $395 \pm 12$ & $408 \pm 12$ & $394 \pm 12$ & $399 \pm 12$ \\
\hline $\mathrm{HDL}_{3 b}$ & $364 \pm 9$ & $343 \pm 9$ & $353 \pm 8 *$ & $329 \pm 8$ * & $336 \pm 9 *$ \\
\hline $\mathrm{HDL}_{3 \mathrm{c}}$ & $128 \pm 5$ & $127 \pm 5$ & $129 \pm 4$ & $118 \pm 4$ & $124 \pm 5$ \\
\hline \multicolumn{6}{|c|}{ Lipoprotein distribution calculated as $\%$ total AUC } \\
\hline TRL & $5.5 \pm 0.4$ & $5.6 \pm 0.6$ & $5.9 \pm 0.4$ & $5.3 \pm 0.4$ & $5.6 \pm 0.6$ \\
\hline $\mathrm{LDL}_{1}$ & $1.5 \pm 0.1$ & $1.2 \pm 0.1$ & $1.3 \pm 0.1$ & $1.3 \pm 0.1$ & $1.5 \pm 0.1$ \\
\hline $\mathrm{LDL}_{2}$ & $7.1 \pm 0.3$ & $6.4 \pm 0.3$ & $6.4 \pm 0.3$ & $6.5 \pm 0.3$ & $7.0 \pm 0.3$ \\
\hline $\mathrm{LDL}_{3}$ & $19.8 \pm 0.9$ & $19.4 \pm 0.8$ & $18.3 \pm 1.0$ & $18.9 \pm 1.0$ & $19.1 \pm 0.7$ \\
\hline $\mathrm{LDL}_{4}$ & $21.8 \pm 0.8$ & $22.5 \pm 0.8$ & $22.4 \pm 0.4$ & $22.4 \pm 0.4$ & $20.8 \pm 0.5^{t, \downarrow, \S}$ \\
\hline
\end{tabular}


Table 4. Cont.

\begin{tabular}{lccccc}
\hline & $\begin{array}{c}\text { Baseline } \\
(\boldsymbol{n}=\mathbf{2 7})\end{array}$ & $\begin{array}{c}\text { HAD } \\
(\boldsymbol{n}=\mathbf{2 7})\end{array}$ & $\begin{array}{c}\text { DASH } \\
(\boldsymbol{n}=\mathbf{2 7})\end{array}$ & $\begin{array}{c}\text { BOLD } \\
(\boldsymbol{n}=\mathbf{2 7})\end{array}$ & $\begin{array}{c}\text { BOLD+ } \\
(\boldsymbol{n}=\mathbf{2 7})\end{array}$ \\
\hline \multicolumn{6}{c}{ Lipoprotein distribution calculated as \% total AUC } \\
$\mathrm{HDL}_{2 \mathrm{~b}}$ & $9.3 \pm 0.4$ & $9.9 \pm 0.4$ & $9.3 \pm 0.3$ & $9.4 \pm 0.3$ & $9.5 \pm 0.5$ \\
$\mathrm{HDL}_{2 \mathrm{a}}$ & $11.2 \pm 0.2$ & $11.3 \pm 0.2$ & $11.3 \pm 0.2$ & $11.7 \pm 0.2$ & $11.8 \pm 0.3$ \\
$\mathrm{HDL}_{3 \mathrm{a}}$ & $10.9 \pm 0.2$ & $10.7 \pm 0.2$ & $11.5 \pm 0.2^{*}+$ & $11.5 \pm 0.2^{*}+$ & $11.5 \pm 0.3^{+}$ \\
$\mathrm{HDL}_{3 \mathrm{~b}}$ & $9.5 \pm 0.2$ & $9.4 \pm 0.3$ & $9.9 \pm 0.2$ & $9.6 \pm 0.2$ & $9.7 \pm 0.3$ \\
$\mathrm{HDL}_{3 \mathrm{c}}$ & $3.4 \pm 0.1$ & $3.5 \pm 0.2$ & $3.6 \pm 0.1$ & $3.5 \pm 0.1$ & $3.6 \pm 0.1$ \\
\hline
\end{tabular}

Data are expressed as mean \pm SEM. $n=27 .{ }^{*} p<0.05$ vs. baseline; ${ }^{\dagger} p<0.05$ vs. HAD $;{ }^{\ddagger} p<0.05$ vs. DASH $;{ }^{\S} p<0.05$ vs. BOLD (Tukey HSD). Skewed data were log transformed before statistical analysis.

\section{4. $\mathrm{LDL}_{4}$ Responsiveness}

To identify possible predictors for individual LDL subclass responses to the HAD, DASH, BOLD and BOLD+ diets, univariate correlations were calculated for the percentage change in $\mathrm{LDL}_{4}$ from baseline with baseline lipid variables, HOMA-IR, and CRP. The results showed that increased baseline HOMA-IR was associated with a less favorable $\mathrm{LDL}_{4}$ response to the HAD $(r=0.36, p=0.065)$, DASH $(r=0.56, p=0.003)$ and BOLD $(r=0.47, p=0.018)$ diets (Table 5$)$. No such clear relationship existed for baseline HOMA-IR with $\mathrm{LDL}_{4}$ response to BOLD+ $(r=0.13, p=0.533)$.

Table 5. Correlations between percent $\mathrm{LDL}_{4}$ change from baseline and baseline lipid, HOMA-IR, and CRP for four test diets.

\begin{tabular}{ccccccccc}
\hline & \multicolumn{2}{c}{ HAD } & \multicolumn{2}{c}{ DASH } & \multicolumn{2}{c}{ BOLD } & \multicolumn{2}{c}{ BOLD+ } \\
\hline & $\boldsymbol{r}$ & $p$ & $\boldsymbol{r}$ & $\boldsymbol{p}$ & $\boldsymbol{r}$ & $\boldsymbol{p}$ & $\boldsymbol{r}$ & $\boldsymbol{p}$ \\
\hline TG & 0.16 & 0.428 & 0.02 & 0.922 & 0.15 & 0.445 & 0.16 & 0.427 \\
TC & 0.16 & 0.412 & 0.27 & 0.161 & 0.13 & 0.502 & 0.21 & 0.300 \\
LDL-C & 0.12 & 0.536 & 0.22 & 0.271 & 0.24 & 0.222 & 0.25 & 0.205 \\
HDL-C & 0.21 & 0.299 & 0.21 & 0.288 & 0.18 & 0.356 & 0.05 & 0.813 \\
HOMA-IR & 0.36 & 0.065 & 0.56 & 0.003 & 0.47 & 0.018 & 0.13 & 0.533 \\
hSCRP & 0.35 & 0.093 & 0.23 & 0.282 & 0.32 & 0.125 & 0.18 & 0.395 \\
\hline
\end{tabular}

Pearson correlation coefficients. For each intervention diet, $n=27$ for lipid variables; $n=25$ for HOMA-IR; $n=24$ for CRP.

To further assess the effect of baseline HOMA-IR on the $\mathrm{LDL}_{4}$ response, participants were grouped into lower and higher baseline HOMA-IR subgroups by median split (< or $\geq 2.73$ ). Baseline subject characteristics following regrouping by median split are shown in Appendix A Table A2. The responses for $\% \mathrm{LDL}_{4}$ for these two groups are shown in Figure 2. The responsiveness to the four diets, expressed as the change in $\% \mathrm{LDL}_{4}$ from baseline, showed no significant difference $(p=0.890)$ in direction among participants with baseline HOMA-IR values $\leq 2.73$; and all showed reductions in $\% \mathrm{LDL}_{4}$. However, within this subgroup, as indicated by unbracketed asterisks, comparison of baseline to individual test diet endpoints demonstrated a significant decrease in $\% \mathrm{LDL}_{4}$ after the consumption of the BOLD $(p=0.003)$ and BOLD+ $(p=0.046)$ diets and no significant decrease after the consumption of the DASH $(p=0.069)$ or HAD $(p>0.1)$ diets. In contrast, directionality of responses to the test diets differed among participants with baseline HOMA-IR $>2.73$. The $\% \mathrm{LDL}_{4}$ decreased in response to the BOLD+ diet, a direction that was significantly different from those for the HAD and DASH diets in the high HOMA-IR group $(p=0.013)$. Indeed, following the consumption of BOLD+ diet, $\% \mathrm{LDL}_{4}$ was numerically lowered in the higher HOMA-IR subgroup $(p<0.1)$, with a magnitude comparable to that observed with all of the diets among subjects with lower HOMA-IR. However, among subjects with higher HOMA-IR values, no trend of decrease in $\% \mathrm{LDL}_{4}$ from baseline was observed following the consumption of other three test diets. 


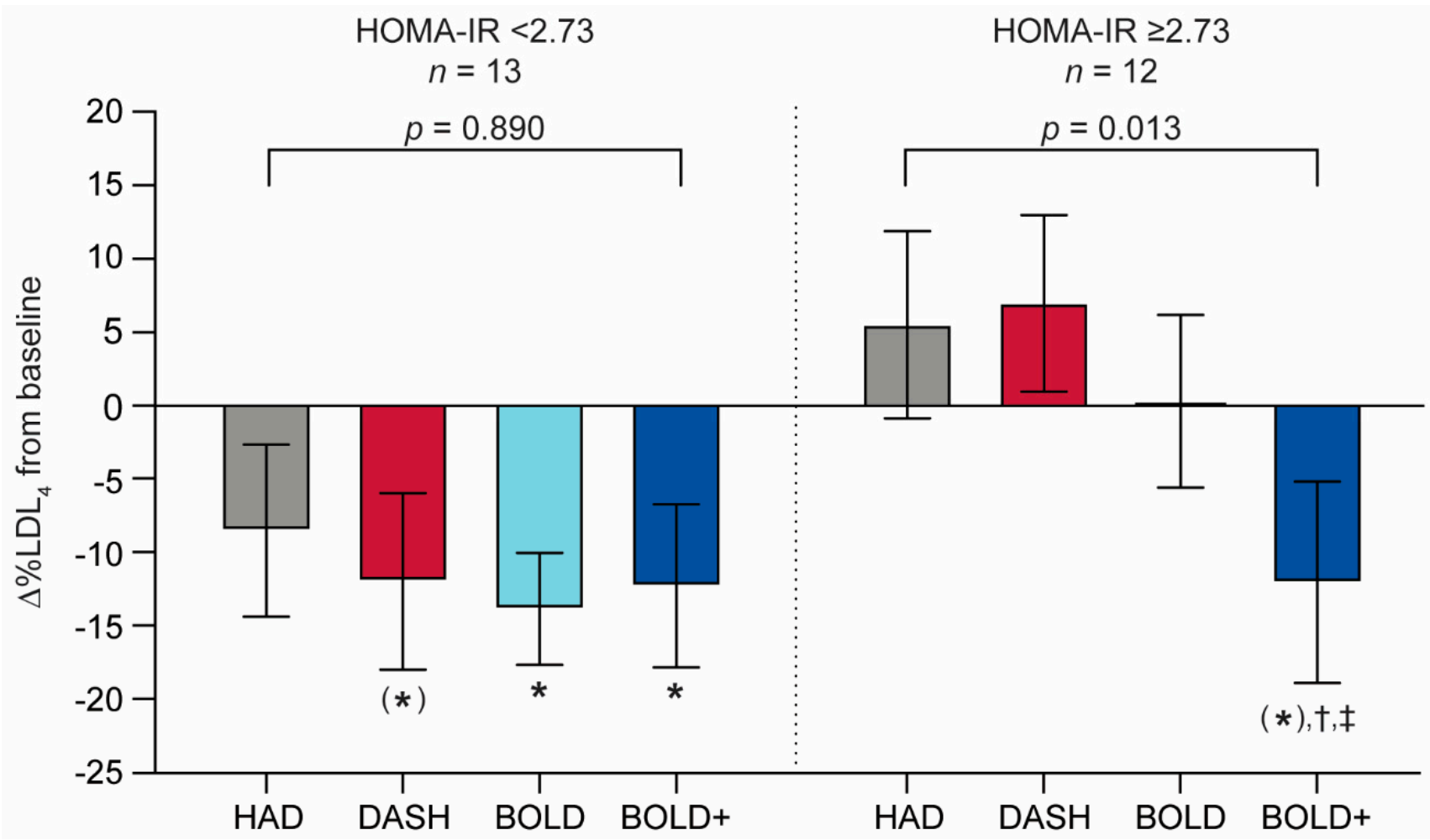

Figure 2. Effects of baseline HOMA-IR on $\mathrm{LDL}_{4}$ response. Lower and higher baseline HOMA-IR subgroups were classified by median split. Values were expressed as mean \pm SEM. Within each subgroup, $\% L_{1} L_{4}$ changes from baseline were compared by repeated measures analysis of covariance with adjustment for age, gender, and BMI. ${ }^{*} p<0.05$ vs. zero; $\left.{ }^{*}\right) p<0.1$ vs. zero. Difference in direction of change within HOMA-IR split group, ${ }^{\dagger} p<0.05$ vs. HAD; ${ }^{\ddagger} p<0.05$ vs. DASH (Tukey HSD). HAD = healthy American diet; DASH = Dietary Approaches to Stop Hypertension; BOLD = Beef in an Optimal Lean Diet; BOLD+ = BOLD plus extra protein.

\section{Discussion}

The participants in the original BOLD study, while being mildly hypercholesterolemic, were normotensive [23] with serum concentrations of TG, glucose, insulin, and HDL-C within normal limits $[15,16,24]$ (and Table 2). Further, subjects maintained their starting bodyweights within $2.2 \mathrm{~kg}$ [22] while consuming the four isocaloric test diets over the $>23$ wk experimental period. Despite the lack of overt evidence of insulin resistance or metabolic syndrome, the ability of three different diets low in total and SFA to reduce the amounts of sdLDL varied in relation with HOMA-IR. Specifically, we found that increasing dietary protein, mainly from $153 \mathrm{~g} /$ day of lean beef in the BOLD+ diet, while decreasing carbohydrate within a DASH-like dietary pattern improved the lipoprotein density profile by decreasing the absolute and proportional abundance of sdLDL, (i.e., $\mathrm{LDL}_{4}$ ) compared to a HAD diet. The degree of improvement was significant in individuals with HOMAIR values $\leq 2.73$; with BOLD+, the difference versus HAD was significant regardless of baseline HOMA-IR. The DASH and BOLD diets also appeared to have beneficial effects on the LDL density profile for some individuals, albeit improvements were less than those observed following the BOLD+ diet. The effects of these two diets appeared to also be influenced by baseline HOMA-IR value. To the best of our knowledge, this is the first study to show that baseline HOMA-IR provides predictive information regarding $\mathrm{LDL}_{4}$ response to a low SFA dietary intervention in normoglycemic individuals.

In the original report from the BOLD study, consumption of the BOLD+ diet reduced LDL cholesterol by $6 \%$ compared to HAD. We now show that cholesterol decrement was associated with a $9.2 \%$ decrease in the sdLDL (i.e., $\mathrm{LDL}_{4}$ ) species that is thought to be the most atherogenic, consistent with a shift toward a less atherogenic distribution of LDL particles $[32,33]$. 
In this analysis, $\mathrm{LDL}_{4}$ particles were defined as those with a density range of $1.044-1.063 \mathrm{~g} / \mathrm{mL}$, which roughly corresponds to LDL particles with a diameter smaller than $24.2 \mathrm{~nm}$ as measured by non-denaturing gradient gel electrophoresis [29]. The prospective Quebec Cardiovascular Study ( $n=2034 \mathrm{men}$ ) found that participants whose absolute or relative cholesterol concentration in LDL particles with a diameter smaller than $25.5 \mathrm{~nm}$ had the strongest association with the risk of ischemic heart disease (relative risk $=4.6$ in men in the third vs. first tertile of the distribution, $p<0.001$ ), after adjustments for other lipid and non-lipid risk factors [34]. These observations suggest that the $9.2 \%$ decrease in sdLDL on the BOLD+ diet might elicit a clinically relevant benefit for cardiovascular disease risk.

Our finding that consistent directional reduction of $\% \mathrm{LDL}_{4}$ (i.e., sdLDL) only occurred in the BOLD+ intervention, not the DASH and BOLD diets, suggests that the BOLD+ diet improvement in LDL density profile was at least partially mediated by the replacement of carbohydrates with protein (mixed source, mainly lean beef), in addition to the effects of SFA reduction. Despite the difference in protein source, this finding is consistent with earlier observations that replacement of carbohydrate by protein in a low SFA diet can improve the lipoprotein profile by shifting LDL distribution to a larger average particle size in overweight and hypertensive subjects [8,35-38]. The underlying mechanism for the decrease in $\mathrm{LDL}_{4}$ by the BOLD+ diet is unknown but may involve alterations in $\mathrm{ApoB}$ metabolism. Higher VLDL ApoB secretion, lower VLDL ApoB fractional conversion rate, as well as a lower LDL ApoB fractional catabolic rate have been observed in healthy men with a predominance of sdLDL (pattern B) (25).

HOMA-IR is a validated measurement of insulin sensitivity in healthy participants with normal glucose tolerance. A value of 1.00 was considered to define healthy normal [39], while a HOMA-IR of $\geq 2.6$ has been previously used as a threshold for classification of low insulin sensitivity [40], which is close to the median value of 2.73 in our sample. Our results are a secondary analysis of samples from the BOLD study [22]. That study did not control for baseline HOMA-IR, and baseline values calculated as a part of the secondary analysis reported for the first time herein ranged from 1.27 to 4.73 . Thus, our comparisons were made on participants whose insulin sensitivity varied from near normal to markedly elevated despite having fasting glucose concentrations within normal limits. The range of values improved our ability to detect the differences in response patterns in this small study.

In this population $\mathrm{HAD}, \mathrm{DASH}, \mathrm{BOLD}$ and BOLD+ produced similar numerical reductions $\% \mathrm{LDL}_{4}$ in the subset with baseline HOMA-IR below the median, although only the BOLD and BOLD+ diets showed statistically significant reductions relative to baseline. In contrast, $\% \mathrm{LDL}_{4}$ responses varied significantly across diet conditions in the subset with higher baseline HOMA-IR, increasing with the HAD and DASH diets, remaining essentially unchanged with the BOLD diet, and decreased with the BOLD+ diet. The BOLD+ diet elicited a reduction in $\% \mathrm{LDL}_{4}$ in the higher HOMA-IR subgroup numerically similar $(p>0.05)$ to that observed with DASH, BOLD or BOLD+ test diets among subjects with lower HOMA-IR. The limited effect of BOLD+ in the higher HOMA-IR may relate to their higher BMI compared to the lower HOMA-IR group ( $24.2 \pm 0.6$ vs. $26.9 \pm 0.9$, $p<0.001$, Appendix A Table A2). Recent studies by some of our group demonstrated that weight loss is the primary driver of metabolic syndrome resolution [41,42].

Changes were observed in additional lipoprotein density subfractions. Consistent with other findings that decreases in large $\mathrm{HDL}_{2}$, i.e., $\mathrm{HDL}_{2 \mathrm{~b}}$, are the most pronounced HDL response in healthy subjects who are shifted to diets low in total fat and SFA [43], is the reduction in $\mathrm{HDL}_{2 \mathrm{~b}}$ following consumption of the DASH and BOLD diets compared to HAD. BOLD+ appeared to modestly reverse the significant decrease in absolute $\mathrm{HDL}_{2 b}$ concentration induced by the DASH and BOLD dietary patterns as amounts were not different from those found on the HAD dietary pattern. Apolipoprotein measurements in the original BOLD study showed that HDL ApoC-III was significantly reduced by BOLD+ compared with HAD [22]. ApoC-III preferentially associates with large $\mathrm{HDL}_{2 b}$ subfrac- 
tions [44] and when enriched in ApoCIII, the anti-inflammatory functionality of $\mathrm{HDL}_{2 b}$ can be compromised [45] and increase risk of cardiovascular disease [46]. A reduction in ApoC-III combined with no change in $\mathrm{HDL}_{2 b}$ amount may suggest an improvement in HDL functionality by the BOLD+ dietary pattern.

The DASH diet emphasized inclusion of complex carbohydrates, plant and lean animal protein sources in the context of dietary pattern rich in fruit, whole grains, and vegetables that is lower in total and SFA. The results of our secondary analysis suggest that recommendations for lifestyle change to alter modifiable risk factors and reduce ischemic heart disease that include reductions in total and SFA intakes should consider increasing protein at the expense of carbohydrate while retaining a fiber intake greater than $30 \mathrm{~g} / \mathrm{day}$ to achieve a BOLD+-type dietary pattern. This dietary pattern seems most appropriate for individuals with elevated HOMA-IR. The lean, unprocessed beef (153 g/day) included in the BOLD+ diet pattern provides a complete protein and is rich in iron, zinc, and the vitamins niacin, $B_{6}$, and $B_{12}$ [47]. These vitamins and minerals are important for antioxidant defenses and tissue repair processes [48,49] and may contribute to the results reported. Daily consumption of this quantity of beef in the context of a BOLD+ dietary pattern and weight maintenance resulted in reduced sdLDL total and fractional amounts in the absence of a reduction in $\mathrm{HDL}_{2 \mathrm{~b}}$.

\section{Conclusions}

In summary, we found that increasing dietary protein, at the expense of carbohydrate, in a low-SFA, heart healthy diet had favorable effects on LDL particle distribution. A low SFA, moderate protein diet including lean beef (BOLD+) improved the LDL density profile by decreasing the absolute and proportional abundance of sdLDL ( $\left.\mathrm{LDL}_{4}\right)$, compared to a higher-SFA HAD, in a mildly hypercholesterolemic study cohort. The results are also consistent with the hypothesis that the $\mathrm{oLDL}_{4}$ response to dietary modifications may differ according to baseline level of insulin resistance as assessed by HOMA-IR. Among subjects with higher baseline HOMA-IR values, the two low-SFA diets did not elicit reductions in $\% \mathrm{LDL}_{4}$ relative to the HAD, whereas levels were reduced with the BOLD+ diet to a degree that was similar to that produced by all three of the low-SFA diets among subjects with lower HOMA-IR values.

Author Contributions: Conceptualization, R.L.W.; methodology, R.L.W. and X.W.; validation, R.L.W. and X.W. (for new density profiling data); formal analysis, X.W.; investigation, R.L.W. and X.W.; resources, P.M.K.-E. and R.L.W.; data curation, R.L.W. and P.M.K.-E.; writing-original draft preparation, X.W.; writing-review and editing, R.L.W., P.M.K.-E., M.A.R., A.M.H.; visualization, X.W.; supervision, R.L.W.; funding acquisition, R.L.W. All authors have read and agreed to the published version of the manuscript.

Funding: This research was funded by the BEEF CHECKOFF PROGRAM, grant number 90413. The APC was funded by AgriLife Research Project \#87380.

Institutional Review Board Statement: The study was conducted according to the Guidelines of the Declaration of Helsinki and approved by the Institutional Review Board at the Pennsylvania State University approved the original experimental protocol, and all subjects provide written informed consent. The trial was registered at clinicaltrials.gov (accessed on 5 September 2021) as NCT00937898.

Informed Consent Statement: Informed consent was obtained from all subjects involved in the original registered trial NCT00937898.

Data Availability Statement: The data presented in this study are available on request from the corresponding author. The data are not publicly available as no appropriate public database is available.

Acknowledgments: Preliminary outcomes reported at the 2014 Experimental Biology meeting in San Diego, California. Wu, X.; Roussell, M.; Smith, S.; Kris-Etherton, P.; Walzem, R. Reduced insulin sensitivity limits reductions in small dense LDL due to lean beef consumption. The FASEB Journal. 2014, 28, 821.814. 
Conflicts of Interest: The authors declare no conflict of interest. The funders had no role in the design of the study; in the collection, analyses, or interpretation of data; in the writing of the manuscript, or in the decision to publish the results.

Appendix A

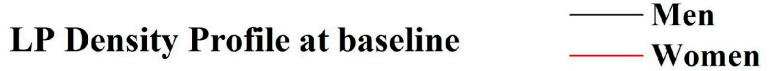

A

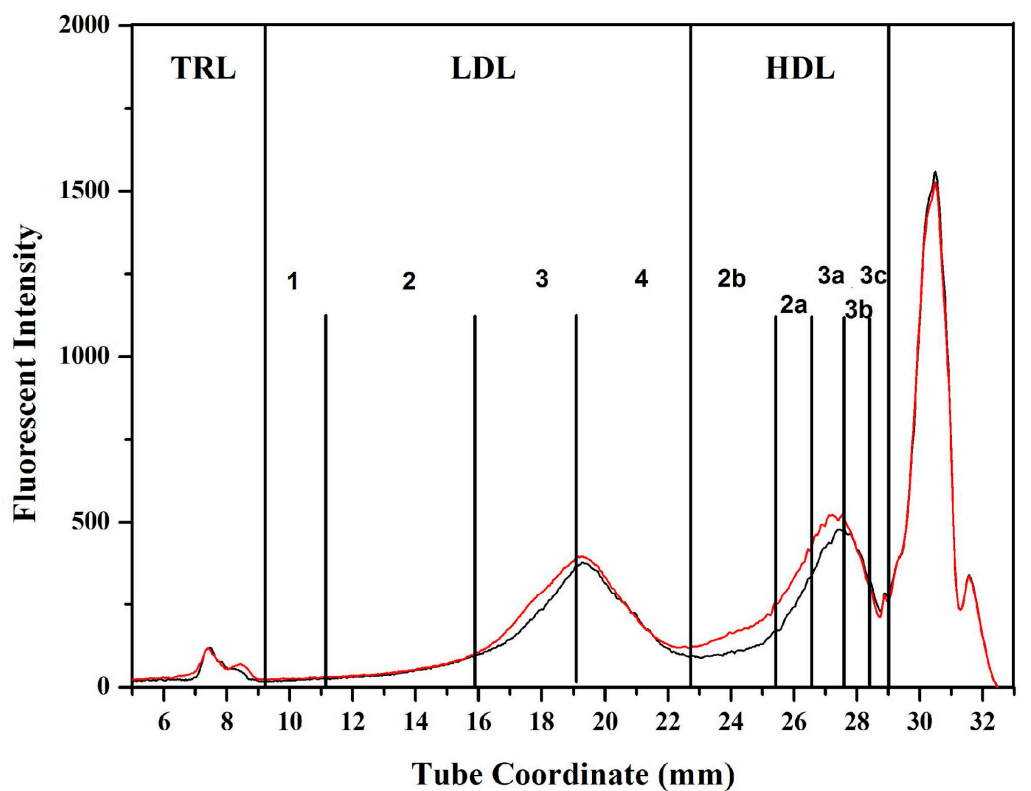

LP Density Profile after BOLD+ - Men Women

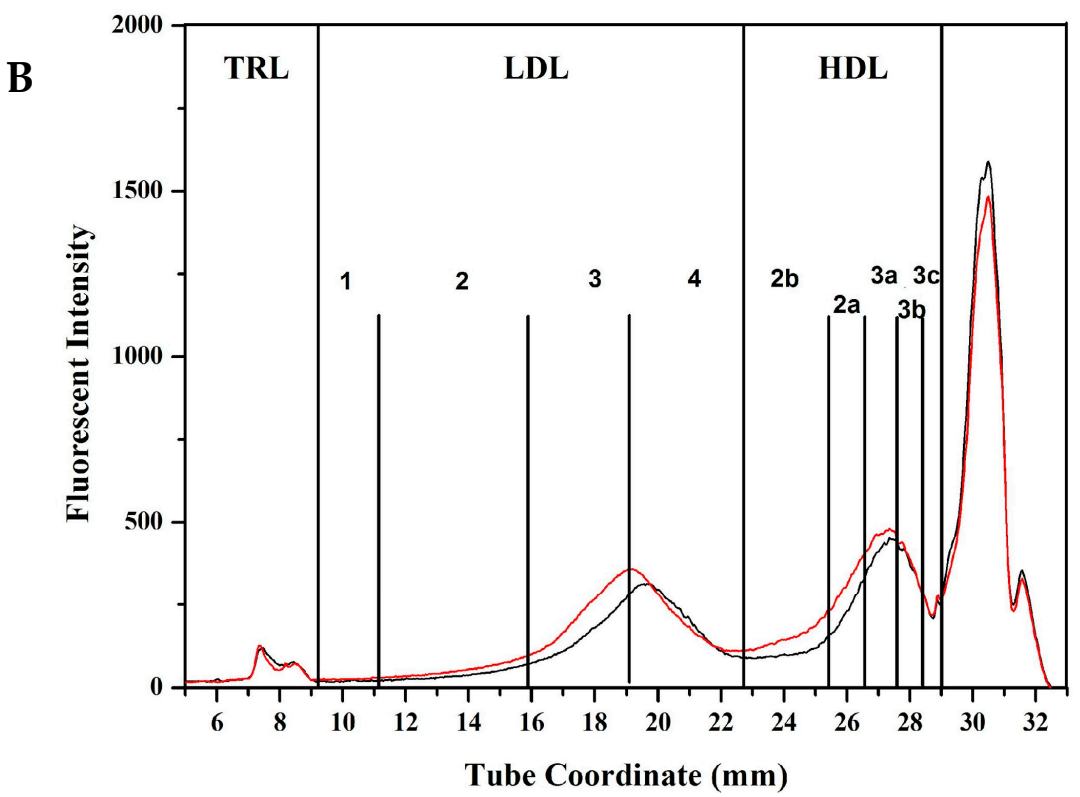

Figure A1. Lipoprotein density profile for male and female participants at baseline (A) and after dietary intervention (B). The BOLD+ diet was used as an example. No significant difference was detected for a gender effect on $\mathrm{LDL}_{4}$ distribution. 


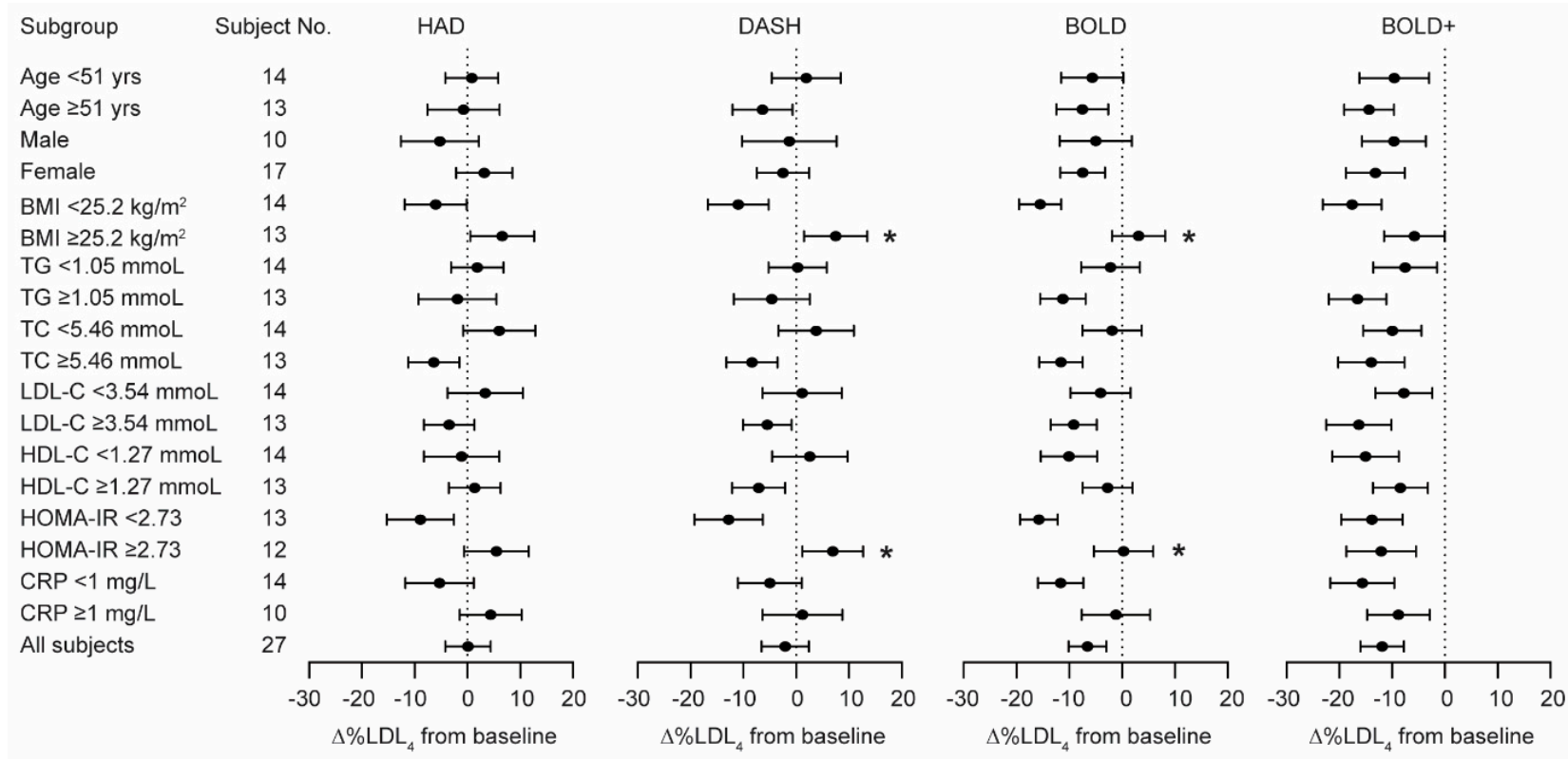

Figure A2. Forest plot. Subgroup analysis for $\mathrm{LDL}_{4}$ response after the 4 test diets. Data are mean $\pm \mathrm{SEM}$. $p<0.05$ between subgroup categories within each diet group as assessed using $t$ test. Skewed data were log transformed before statistical analysis.

Table A1. Reproducibility for intra- and inter- assays.

\begin{tabular}{|c|c|c|c|c|c|c|}
\hline \multirow[b]{2}{*}{ TG } & \multicolumn{3}{|c|}{ Intra-Assay $(n=3)$} & \multicolumn{3}{|c|}{ Inter-Assay $(n=5)$} \\
\hline & Low & Medium & High & Low & Medium & High \\
\hline \multicolumn{7}{|c|}{ Lipoprotein distribution calculated as absolute AUC } \\
\hline TRL & 18.6 & 13.9 & 14.9 & 19.8 & 11.3 & 10.6 \\
\hline $\mathrm{LDL}_{1}$ & 33.6 & 21.8 & 12.9 & 32.6 & 21.7 & 8.2 \\
\hline $\mathrm{LDL}_{2}$ & 10.1 & 5.7 & 8.6 & 11.3 & 6.8 & 4.1 \\
\hline $\mathrm{LDL}_{3}$ & 2.4 & 5.1 & 8.0 & 2.9 & 4.4 & 4.7 \\
\hline $\mathrm{LDL}_{4}$ & 6.2 & 9.4 & 8.4 & 5.4 & 5.1 & 7.6 \\
\hline $\mathrm{HDL}_{2 b}$ & 1.5 & 4.6 & 6.4 & 0.2 & 1.4 & 2.9 \\
\hline $\mathrm{HDL}_{2 \mathrm{a}}$ & 3.4 & 4.7 & 6.1 & 2.0 & 1.5 & 0.4 \\
\hline $\mathrm{HDL}_{3 \mathrm{a}}$ & 3.2 & 4.6 & 4.4 & 1.9 & 1.7 & 1.9 \\
\hline $\mathrm{HDL}_{3 b}$ & 4.3 & 3.3 & 1.8 & 3.0 & 3.4 & 6.8 \\
\hline $\mathrm{HDL}_{3 \mathrm{c}}$ & 13.9 & 10.8 & 9.3 & 14.4 & 9.5 & 13.4 \\
\hline Average & 9.7 & 8.4 & 8.1 & 9.3 & 6.7 & 6.1 \\
\hline \multicolumn{7}{|c|}{ Lipoprotein distribution calculated as \% total AUC } \\
\hline TRL & 10.8 & 8.8 & 22.9 & 9.6 & 8.8 & 19.1 \\
\hline $\mathrm{LDL}_{1}$ & 21.9 & 19.2 & 12.2 & 21.4 & 18.1 & 11.2 \\
\hline $\mathrm{LDL}_{2}$ & 5.9 & 4.3 & 8.0 & 4.4 & 3.4 & 4.1 \\
\hline $\mathrm{LDL}_{3}$ & 5.0 & 5.6 & 3.9 & 5.1 & 4.9 & 5.5 \\
\hline $\mathrm{LDL}_{4}$ & 4.3 & 4.3 & 5.9 & 3.4 & 3.5 & 4.5 \\
\hline $\mathrm{HDL}_{2 b}$ & 3.2 & 1.9 & 7.2 & 2.2 & 1.9 & 5.9 \\
\hline $\mathrm{HDL}_{2 \mathrm{a}}$ & 8.7 & 4.2 & 3.8 & 7.7 & 5.0 & 5.8 \\
\hline $\mathrm{HDL}_{3 a}$ & 6.1 & 2.3 & 4.3 & 5.5 & 3.4 & 3.8 \\
\hline $\mathrm{HDL}_{3 \mathrm{~b}}$ & 4.6 & 2.0 & 7.3 & 4.2 & 0.8 & 4.0 \\
\hline $\mathrm{HDL}_{3 \mathrm{c}}$ & 11.7 & 8.4 & 9.9 & 9.9 & 8.2 & 6.1 \\
\hline Average & 8.2 & 6.1 & 8.5 & 7.3 & 5.8 & 7.0 \\
\hline
\end{tabular}

Coefficients of variation for intra-assay and inter-assay. Low TG $=0.63 \mathrm{mmol} / \mathrm{L}$, medium TG $=1.15 \mathrm{mmol} / \mathrm{L}$, high TG $=2.25 \mathrm{mmol} / \mathrm{L}$. 
Table A2. Subject characteristics following reclassification according to HOMA-IR median split.

\begin{tabular}{lcc}
\hline & Lower HOMA-IR & Higher HOMA-IR \\
\hline HOMA-IR & $2.1 \pm 0.1$ & $3.4 \pm 0.2 *$ \\
SEX, M:F, $n$ & $5: 8$ & $5: 9$ \\
Age (years) & $51.1 \pm 2.3$ & $50.8 \pm 2.5$ \\
BMI & $24.2 \pm 0.6$ & $26.9 \pm 0.9 *$ \\
Triacylglycerol (mg/dL) & $86.1 \pm 5.9$ & $106.2 \pm 8.2$ \\
Total cholesterol (mg/dL) & $219.8 \pm 7.6$ & $207.2 \pm 8.5$ \\
LDL cholesterol (mg/dL) & $145.3 \pm 5.5$ & $137.2 \pm 7.2$ \\
HDL cholesterol (mg/dL) & $57.3 \pm 4.2$ & $48.9 \pm 3.3$ \\
hsCRP (mg/L) & $1.1 \pm 0.3$ & $1.6 \pm 0.4$ \\
\hline
\end{tabular}

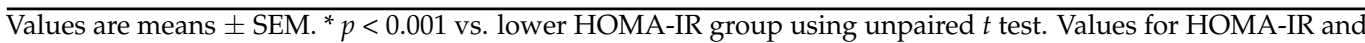
hsCRP were log transformed for analysis.

\section{References}

1. La Belle, M.; Krauss, R.M. Differences in carbohydrate content of low density lipoproteins associated with low density lipoprotein subclass patterns. J. Lipid Res. 1990, 31, 1577-1588. [CrossRef]

2. Chait, A.; Brazg, R.L.; Tribble, D.L.; Krauss, R.M. Susceptibility of small, dense, low-density lipoproteins to oxidative modification in subjects with the atherogenic lipoprotein phenotype, pattern B. Am. J. Med. 1993, 94, 350-356. [CrossRef]

3. Nigon, F.; Lesnik, P.; Rouis, M.; Chapman, M.J. Discrete subspecies of human low density lipoproteins are heterogeneous in their interaction with the cellular LDL receptor. J. Lipid Res. 1991, 32, 1741-1753. [CrossRef]

4. Borén, J.; Chapman, M.J.; Krauss, R.M.; Packard, C.J.; Bentzon, J.F.; Binder, C.J.; Daemen, M.J.; Demer, L.L.; Hegele, R.A.; Nicholls, S.J.; et al. Low-density lipoproteins cause atherosclerotic cardiovascular disease: Pathophysiological, genetic, and therapeutic insights: A consensus statement from the European Atherosclerosis Society Consensus Panel. Eur. Heart J. 2020, 41, 2313-2330. [CrossRef]

5. Dreon, D.M.; Fernstrom, H.A.; Miller, B.; Krauss, R.M. Apolipoprotein-E isoform phenotype and LDL subclass response to a reduced-fat diet. Arterioscler. Thromb. Vasc. Biol. 1995, 15, 105-111. [CrossRef]

6. Dreon, D.M.; Fernstrom, H.A.; Williams, P.T.; Krauss, R.M. Reduced LDL particle size in children consuming a very-low-fat diet is related to parental LDL-subclass patterns. Am. J. Clin. Nutr. 2000, 71, 1611-1616. [CrossRef]

7. KasimKarakas, S.E.; Lane, E.; Almario, R.; Mueller, W.; Walzem, R. Effects of dietary fat restriction on particle size of plasma lipoproteins in postmenopausal women. Metab. Clin. Exp. 1997, 46, 431-436. [CrossRef]

8. Krauss, R.M.; Blanche, P.J.; Rawlings, R.S.; Fernstrom, H.S.; Williams, P.T. Separate effects of reduced carbohydrate intake and weight loss on atherogenic dyslipidemia. Am. J. Clin. Nutr. 2006, 83, 1025-1031. [CrossRef]

9. Krauss, R.M.; Dreon, D.M. Low-density-lipoprotein subclasses and response to a low-fat diet in healthy-men. Am. J. Clin. Nutr. 1995, 62, S478-S487. [CrossRef]

10. Chapman, M.J.; Laplaud, P.M.; Luc, G.; Forgez, P.; Bruckert, E.; Goulinet, S.; Lagrange, D. Further resolution of the low density lipoprotein spectrum in normal human plasma: Physicochemical characteristics of discrete subspecies separated by density gradient ultracentrifugation. J. Lipid Res. 1988, 29, 442-458. [CrossRef]

11. Nunn, A.V.; Bell, J.D.; Guy, G.W. Lifestyle-induced metabolic inflexibility and accelerated ageing syndrome: Insulin resistance, friend or foe? Nutr. Metab. 2009, 6, 16. [CrossRef]

12. Gower, B.A.; Bergman, R.; Stefanovski, D.; Darnell, B.; Ovalle, F.; Fisher, G.; Sweatt, S.K.; Resuehr, H.S.; Pelkman, C. Baseline insulin sensitivity affects response to high-amylose maize resistant starch in women: A randomized, controlled trial. Nutr. Metab. 2016, 13, 2. [CrossRef]

13. Lin, D.; Qi, Y.; Huang, C.; Wu, M.; Wang, C.; Li, F.; Yang, C.; Yan, L.; Ren, M.; Sun, K. Associations of lipid parameters with insulin resistance and diabetes: A population-based study. Clin. Nutr. 2018, 37, 1423-1429. [CrossRef]

14. Palomba, S.; Falbo, A.; Chiossi, G.; Muscogiuri, G.; Fornaciari, E.; Orio, F.; Tolino, A.; Colao, A.; La Sala, G.B.; Zullo, F. Lipid profile in nonobese pregnant women with polycystic ovary syndrome: A prospective controlled clinical study. Steroids 2014, 88, 36-43. [CrossRef]

15. American Diabetes, A. 2. Classification and diagnosis of diabetes. Diabetes Care 2016, 39 (Suppl. 1), S13-S22. [CrossRef]

16. Ferrannini, E.; Mari, A. Physiology of insulin secretion. In Williams Textbook of Endocrinology, 14th ed.; Shlomo Melmed, S., Auchus, R.J., Goldfine, A.B., Koenig, R.J., Rosen, C.J., Eds.; Elsevier: Philadelphia, PA, USA, 2020; pp. 1338-1348.e33.

17. Appel, L.J.; Moore, T.J.; Obarzanek, E.; Vollmer, W.M.; Svetkey, L.P.; Sacks, F.M.; Bray, G.A.; Vogt, T.M.; Cutler, J.A.; Windhauser, M.M.; et al. A clinical trial of the effects of dietary patterns on blood pressure. DASH Collaborative Research Group. N. Engl. J. Med. 1997, 336, 1117-1124. [CrossRef]

18. Conlin, P.R.; Chow, D.; Miller, E.R.; Svetkey, L.P.; Lin, P.H.; Harsha, D.W.; Moore, T.J.; Sacks, F.M.; Appel, L.J.; Grp, D.R. The effect of dietary patterns on blood pressure control in hypertensive patients: Results from the Dietary Approaches to Stop Hypertension (DASH) trial. Am. J. Hypertens. 2000, 13, 949-955. [CrossRef] 
19. Kaluza, J.; Akesson, A.; Wolk, A. Processed and unprocessed red meat consumption and risk of heart failure prospective study of men. Circ. Heart. Fail. 2014, 7, 552-557. [CrossRef]

20. Maki, K.C.; Slavin, J.L.; Rains, T.M.; Kris-Etherton, P.M. Limitations of observational evidence: Implications for evidence-based dietary recommendations. Adv. Nutr. 2014, 5, 7-15. [CrossRef]

21. Rohrmann, S.; Overvad, K.; Bueno-De-Mesquita, H.B.; Jakobsen, M.U.; Egeberg, R.; Tjonneland, A.; Nailler, L.; Boutron-Ruault, M.C.; Clavel-Chapelon, F.; Krogh, V.; et al. Meat consumption and mortality-results from the European prospective investigation into cancer and nutrition. BMC Med. 2013, 11, 63. [CrossRef]

22. Roussell, M.A.; Hill, A.M.; Gaugler, T.L.; West, S.G.; Heuvel, J.P.; Alaupovic, P.; Gillies, P.J.; Kris-Etherton, P.M. Beef in an optimal lean diet study: Effects on lipids, lipoproteins, and apolipoproteins. Am. J. Clin. Nutr. 2012, 95, 9-16. [CrossRef] [PubMed]

23. Roussell, M.A.; Hill, A.M.; Gaugler, T.L.; West, S.G.; Ulbrecht, J.S.; Vanden Heuvel, J.P.; Gillies, P.J.; Kris-Etherton, P.M. Effects of a DASH-like diet containing lean beef on vascular health. J. Hum. Hypertens. 2014, 28, 600-605. [CrossRef] [PubMed]

24. Kwiterovich, P.O. Clinical relevance of the biochemical, metabolic, and genetic factors that influence low-density lipoprotein heterogeneity. Am. J. Cardiol. 2002, 90, 30i-47i. [CrossRef]

25. Morgan, C.R.; Lazarow, A. Immunoassay of Insulin Using a Two-Antibody System. Proc. Soc. Exp. Biol. Med. 1962, 110, $29-32$. [CrossRef]

26. Kraus, R.M.; Stallings, H.W.; Yeager, R.C.; Gavin, T.P. Circulating plasma VEGF response to exercise in sedentary and endurancetrained men. J. Appl. Physiol. 2004, 96, 1445-1450. [CrossRef]

27. Wallace, T.M.; Levy, J.C.; Matthews, D.R. Use and abuse of HOMA modeling. Diabetes Care 2004, 27, 1487-1495. [CrossRef]

28. Heinze, C.R.; Hawkins, M.G.; Gillies, L.A.; Wu, X.; Walzem, R.L.; German, J.B.; Klasing, K.C. Effect of dietary omega-3 fatty acids on red blood cell lipid composition and plasma metabolites in the cockatiel, Nymphicus hollandicus. J. Anim. Sci. 2012, 90, 3068-3079. [CrossRef]

29. Berneis, K.K.; Krauss, R.M. Metabolic origins and clinical significance of LDL heterogeneity. J. Lipid Res. 2002, 43, 1363-1379. [CrossRef]

30. Guerin, M.; Bruckert, E.; Dolphin, P.J.; Turpin, G.; Chapman, M.J. Fenofibrate reduces plasma cholesteryl ester transfer from HDL to VLDL and normalizes the atherogenic, dense LDL profile in combined hyperlipidemia. Arterioscler. Thromb. Vasc. Biol. 1996, 16, 763-772. [CrossRef]

31. Orsoni, A.; Saheb, S.; Levels, J.H.; Dallinga-Thie, G.; Atassi, M.; Bittar, R.; Robillard, P.; Bruckert, E.; Kontush, A.; Carrie, A.; et al. LDL-apheresis depletes apoE-HDL and pre-beta1-HDL in familial hypercholesterolemia: Relevance to atheroprotection. J. Lipid Res. 2011, 52, 2304-2313. [CrossRef]

32. Veniant, M.M.; Sullivan, M.A.; Kim, S.K.; Ambroziak, P.; Chu, A.; Wilson, M.D.; Hellerstein, M.K.; Rudel, L.L.; Walzem, R.L.; Young, S.G. Defining the atherogenicity of large and small lipoproteins containing apolipoprotein B100. J. Clin. Investig. 2000, 106, 1501-1510. [CrossRef]

33. Lamarche, B.; St-Pierre, A.C.; Ruel, I.L.; Cantin, B.; Dagenais, G.R.; Despres, J.P. A prospective, population-based study of low density lipoprotein particle size as a risk factor for ischemic heart disease in men. Can. J. Cardiol. 2001, 17, 859-865.

34. St-Pierre, A.C.; Ruel, I.L.; Cantin, B.; Dagenais, G.R.; Bernard, P.M.; Despres, J.P.; Lamarche, B. Comparison of various electrophoretic characteristics of LDL particles and their relationship to the risk of ischemic heart disease. Circulation 2001, 104, 2295-2299. [CrossRef]

35. Dumesnil, J.G.; Turgeon, J.; Tremblay, A.; Poirier, P.; Gilbert, M.; Gagnon, L.; St-Pierre, S.; Garneau, C.; Lemieux, I.; Pascot, A.; et al. Effect of a low-glycaemic index-low-fat-high protein diet on the atherogenic metabolic risk profile of abdominally obese men. Br. J. Nutr. 2001, 86, 557-568. [CrossRef] [PubMed]

36. Furtado, J.D.; Campos, H.; Appel, L.J.; Miller, E.R.; Laranjo, N.; Carey, V.J.; Sacks, F.M. Effect of protein, unsaturated fat, and carbohydrate intakes on plasma apolipoprotein B and VLDL and LDL containing apolipoprotein C-III: Results from the OmniHeart Trial. Am. J. Clin. Nutr. 2008, 87, 1623-1630. [CrossRef] [PubMed]

37. Lefevre, M.; Champagne, C.M.; Tulley, R.T.; Rood, J.C.; Most, M.M. Individual variability in cardiovascular disease risk factor responses to low-fat and low-saturated-fat diets in men: Body mass index, adiposity, and insulin resistance predict changes in LDL cholesterol. Am. J. Clin. Nutr. 2005, 82, 957-963. [CrossRef]

38. Maki, K.C.; Palacios, O.M.; Lindner, E.; Nieman, K.M.; Bell, M.; Sorce, J. Replacement of refined starches and Added sugars with egg protein and unsaturated fats increases insulin sensitivity and lowers triglycerides in overweight or obese adults with elevated triglycerides. J. Nutr. 2017, 147, 1267-1274. [CrossRef] [PubMed]

39. Muniyappa, R.; Lee, S.; Chen, H.; Quon, M.J. Current approaches for assessing insulin sensitivity and resistance in vivo: Advantages, limitations, and appropriate usage. Am. J. Physiol. Endocrinol. Metab. 2008, 294, E15-E26. [CrossRef]

40. Ascaso, J.F.; Pardo, S.; Real, J.T.; Lorente, R.I.; Priego, A.; Carmena, R. Diagnosing insulin resistance by simple quantitative methods in subjects with normal glucose metabolism. Diabetes Care 2003, 26, 3320-3325. [CrossRef] [PubMed]

41. Pedersen, L.R.; Olsen, R.H.; Anholm, C.; Walzem, R.L.; Fenger, M.; Eugen-Olsen, J.; Haugaard, S.B.; Prescott, E. Weight loss is superior to exercise in improving the atherogenic lipid profile in a sedentary, overweight population with stable coronary artery disease: A randomized trial. Atherosclerosis 2016, 246, 221-228. [CrossRef]

42. Hill, A.M.; Harris Jackson, K.A.; Roussell, M.A.; West, S.G.; Kris-Etherton, P.M. Type and amount of dietary protein in the treatment of metabolic syndrome: A randomized controlled trial. Am. J. Clin. Nutr. 2015, 102, 757-770. [CrossRef] 
43. Berglund, L.; Oliver, E.H.; Fontanez, N.; Holleran, S.; Matthews, K.; Roheim, P.S.; Ginsberg, H.N.; Ramakrishman, R.; Lefevre, M.; Investigators, D. HDL-subpopulation patterns in response to reductions in dietary total and saturated fat intakes in healthy subjects. Am. J. Clin. Nutr. 1999, 70, 992-1000.

44. Davidson, W.S.; Silva, R.A.; Chantepie, S.; Lagor, W.R.; Chapman, M.J.; Kontush, A. Proteomic analysis of defined HDL subpopulations reveals particle-specific protein clusters: Relevance to antioxidative function. Arterioscler. Thromb. Vasc. Biol. 2009, 29, 870-876. [CrossRef] [PubMed]

45. Kawakami, A.; Aikawa, M.; Libby, P.; Alcaide, P.; Luscinskas, F.W.; Sacks, F.M. Apolipoprotein CIII in apolipoprotein B lipoproteins enhances the adhesion of human monocytic cells to endothelial cells. Circulation 2006, 113, 691-700. [CrossRef] [PubMed]

46. Jensen, M.K.; Rimm, E.B.; Furtado, J.D.; Sacks, F.M. Apolipoprotein C-III as a potential modulator of the association between HDL-cholesterol and incident coronary heart disease. J. Am. Heart. Assoc. 2012, 1, jah3-e000232. [CrossRef] [PubMed]

47. United States Department of Agriculture, A.R.S. FoodData Central; United States Department of Agriculture: Washington, DC, USA, 2019.

48. Combs, G.F.; McClung, J.P. Vitamins: Fundamental Aspects in Nutrition and Health, 5th ed.; Academic Press: Cambridge, UK, 2017. [CrossRef]

49. O’Dell, B.L.; Sunde, R.A. Handbook of Nutritionaly Essential Mineral Elements; Marcel Dekker Inc.: New York, NY, USA, 1997; Volume 1, p. 692. 